\title{
RESEARCHES ON THE PERFUSED HEART \\ SOME ACTIONS OF ADRENIN TOGETHER WITH SOME REMARKS ON ADDISON'S DISEASE
}

\author{
BY W. BURRIDGE
}

(From the Physiological Laboratory, Oxford, and Guy's Hospital, London)

With Plates 21-25

THE well-known researches of Langley (18), Brodie and Dixon (4), Elliott (13), and others, have shown that adrenin is a drug capable of exciting in an organ the activities normally associated with excitation of sympathetic nerves. It has also been suggested by Howell and Duke (17) that the sympathetic nerves of the heart produce their effects by releasing calcium salts at their termination. The theory is based on their discovery that a sudden increase in the calcium content of a solution perfusing the heart may be followed by effects simulating sympathetic stimulation.

Certain objections may be raised against this theory. Inasmuch as the addition of calcium to the perfusing solution gives effects simulating sympathetic stimulation, it would follow that such perfused calcium salts penetrate to those structures where, according to the theory, excitation of sympathetic nerves releases calcium, and also that such released calcium can diffuse into the blood stream. But there is good evidence that sympathetic nerves exercise a tonic influence on the heart, and it is difficult to imagine them continuously releasing calcium salts. Moreover, the stimulating action which follows an increase of the calcium content of a perfusing solution disappears almost immediately after the calcium content is reduced once more to normal, and in addition a depressed state of the heart is left behind (6); whereas the effects following stimulation of sympathetic nerves persist some time after cessation of stimulation and we have not the depression.

Underlying this suggestion of Howell and Duke appears to be an assumption of a constancy of action produced by a given tension of calcium. I have given an abundance of evidence here and elsewhere (5-10), however, indicating that such a view is untenable. The magnitude of the cardiac response to calcium is an ever-varying value, and according to the nature of that response so calcium produces different results. This knowledge enables the suggestion to be 
put forward that during sympathetic stimulation there is a change in the response of the heart to calcium, the change being that of an augmentation. Such a mechanism presents sympathetic stimulation as inducing a change in the response of the heart to its immediate and constant environment rather than as an autogenous change of environment reacting on the heart itself, as suggested by Howell and Duke.

Some evidence for this suggestion is given in the present paper, together with an account of other experiments which, it is believed, throw some light on the pathology of Addison's disease. The actual material of the paper will be found eapable of a rough division into three main parts. In the first of these certain relations between adrenin and calcium salts are considered, and they show that adrenin may be regarded as a device enabling the organism to carry out certain functions in presence of less calcium than would otherwise be necessary. Some possible advantages of such a system have been discussed in an earlier paper (10).

The second portion of this paper deals with the action of adrenin whereby it enables cardiac activity to be maintained over long periods of time when the heart is perfused with solutions showing a decided lack of 'balance' between their' inorganic elements. It is shown that the 'balance' between the inorganic elements of a perfusing solution acquires a secondary importance, so far as the mere maintenance of cardiac activity is concerned, when traces of adrenin are also present.

The third portion deals with certain changes constantly observed when Ringer's solution is substituted for blood as a perfusing medium, and with their interpretation. The latter provides also a convenient explanation of the origin of certain symptoms of Addison's disease.

\section{Method of Experiment.}

The experiments bave been carried out on the hearts of Rana temporaria perfused $i n$ situ through a cannula inserted into the inferior vena cava, as already described.

The adrenin preparations used were the adrenalin hydrochloride solution prepared by the Parke Davis Co., hemisine and tabloid suprarenal extract prepared by Messrs. Burroughs and Wellcome.

\section{Results.}

The fluctuations in the level of the summation line which follow the addition of adrenin to a solution perfusing the faradized heart may be deseribed first. An illustration of the change is given in Plate 21, Fig. 1.

On faradizing a heart perfused with Ringer's solution the heart enters 
into a fluctuating semi-contracted state. The contractions evoked by the faradism usually start from a line, the summation line, situated at a higher level than the line, the base line, whence start the spontaneous contractions (8).

A moderate dose of adrenin first quickly depressed and then gradually raised this summation line; a strong dose (1 pt. in 25,000, say) depressed it; the weaker doses ( 1 in $1,000,000$, say) had no particular action, or slightly raised it.

An experiment such as that shown in the figure above is quickly and easily performed and has the great advantage of providing a directing post for subsequent work. Thus, having obtained that primary depression shown in the figure, experience bas shown me there will be a 'surface' (7) antagonism between calcium and the substance producing that depression. But to show that antagonism it may be necessary to make a selection of both perfusing solution and of concentration of drug. An appropriate selection is shown in Fig. 2.

From the arrow on the left of the tracing to the extreme right the perfusing solutions contained 1 part in 25,000 of the Parke Davis adrenalin hydrochloride. On the extreme left the heart was perfused with a Ringer in which the calcium concentration was represented by one-fifth saturation with the dibasic phosphate of calcium. The condition of the heart at the commencement of the experiment was such that it was beating well on this solution. Incidentally, it may be remarked that a capacity for beating well on that solution, apart from experimental interference with drugs, may be taken as good evidence that the heart was 'fresh'. The perfused heart quickly loses its response to calcium, sufficient to permit of good activity on that solution.

After addition of the adrenalin to that solution the beats of the heart quickly fell away, but were restored again when the calcium content of the solution was increased to full saturation. The experiment was repeated three times, the degree of failure being permitted to proceed further on each new occasion, until on the third occasion the beats became almost imperceptible.

A few remarks on the two solutions used above would not be out of place here. The solution of lower calcium concentration was not one that would conventionally be regarded as a 'good' perfusing solution. The usual test of a good saline perfusing solution is to note how long a heart beats when perfused with it. The longer the heart maintains activity, the better the perfusing solution. The conclusion is so obvious as to be almost a self-evident proposition. Yet it will be found that different observers recommend different solutions, and that not a few as a preliminary to other work find out for themselves what is the 'best' solution.

If, however, the experimenter considers that an inorganic saline perfusing solution is a makeshift device, as to which evidence is given later, a different course is open to him. My own method is to use a number of different perfusing solutions constant in composition except as regards the calcium content, to ascertain the maximum contractibility of the heart, and to test its 'state' at 
intervals. The advantages of the method as they appear to me may be brought out by a mechanical metaphor. The experimenter works with a machine of known maximum horse-power, he knows what proportion of that maximum is being given out at a particular moment, and he knows the state of the throttle required to elicit it, whereas the other method provides him with a machine of unknown horse-power and a fixed throttle. With the latter method, although he may be conscious that the machine at a particular moment is not working as well as it did at some previous time, he cannot properly appreciate that depression.

In the case above, the sole use of the solution of higher calcium content, the 'good ' perfusing solution, would have revealed a depression of the summation line in the faradized heart, but no corresponding depression of the spontaneous contractions.

The state of the heart at the time the experiment was made was also of importance. The more responsive the heart was to calcium, the less readily was it depressed by adrenin. There are, thus, three factors in this depressing action of adrenin, viz. : (1) The concentration of the drug; (2) the concentration of the calcium in the perfusing solution; and (3) the condition of the heart at the time of the experiment.

This depressive action of adrenin was exhibited in varying degrees according to the particular combination present of the conditions determining its appearance. What is possibly of importance in connexion with it is the fact that actual concentration of drug is only one of the three factors concerned. The higher the concentration of drug used the more likely is it to produce this depression, so that it is a drug which should not be pushed too hard. The experiments also indicate the possibility that a dose of the drug which would stimulate a normal individual might have untoward consequences in an emergency.

An inhibitory action of suprarenal extract was noticed by Langley (18) and by Oliver and Schäfer (21), the first of these also observing the action after section of the vagi. The depressive action of adrenin described above shows many resemblances to inbibition not only when the drug was actually present, but also in the 'rebound' to augmented activity observed subsequently and described below. It is, I think, a truly inhibitory action, but its discussion is, perhaps, best left to a later period, where it can be considered in connexion with the augmenting action of adrenin to be immediately described.

The augmenting, or sympathomimetic, action (2) of adrenin corresponds to the secondary raising of the summation line observed when adrenin is perfused through the faradized heart. But here again it was a question of getting the appropriate combination of drug and perfusing solution for its proper manifestation. In my experiments the augmenting action of adrenin followed the depressing action whenever the latter was also shown. One limit of experimenting was reached when the adrenin was used in quantities sufficient to stop the heart in diastole such bas already been described. In such cases when the adrenin was washed ont of the heart the latter not only recovered its ability to 
beat spontaneously, but it now beat much better than was the case before the exposure to adrenin. The beats were now quicker in rate and of increased amplitude as compared with what they had been before treatment with the adrenin, the improvement persisting some little time, after which the heart gradually returned to the normal.

The relatively large dose of adrenin has, then, a twofold action on the heart. There is a depressing action which comes on and goes away with addition to and abstraction from the perfusing solution of the adrenin; and there is an exalting action which depends on previous contact of drug and heart, but does not then require the presence of the drug in the perfusing solution for its immediate manifestation. From what has been stated in previous papers the depressing action of adrenin is a 'surface' action; and its favouring influence on cardiac activity, a 'deep' action (7-10).

An examination of hearts during the period of exalted cardiac activity brought about by adrenin showed that during this period the heart had a greatly inereased responsiveness to calcium as compared with what it had before treatment with the adrenin. Both these actions are very well shown in Fig. 3.

It will be found instructive to compare Fig. 3 with Fig. 2 . In the latter the experiment shown was performed on a heart capable of beating well on a Ringer with a calcium content of one-fifth saturation with the dibasic phosphate. In the experiment now considered, the heart, to beat well on a similar solution, required full saturation with the calcium salt as shown by the contractions recorded on the extreme left of the tracing, for on reducing the calcium content to one-fifth saturation the beats became very small. When equilibrium to the new solution had just been established adrenalin hydrochloride was added to it ( 1 in 250,000).

Following the addition of the adrenalin to the solution there was a wellmarked primary depression succeeded by a much greater augmentation. The resemblance of that depression to inhibition is also well shown. Later the adrenalin was removed from the perfnsing solution, but it will be observed that the improvement persisted, and then the beats gradually fell away again. About twenty minutes elapsed between removal of the adrenalin and the return of the heart to the condition existing before exposure to the drug.

The particular experiment illustrated above was one of a series performed on that heart. In that series the heart was treated on each new occasion with a smaller dose of adrenalin than the one going before. The experiments showed that a dilution of the adrenalin of 1 in $10,000,000$ produced practically the same amount of augmentation as that shown above. At a dilution of 1 in 50,000,000 the augmentation was about one-half that shown. But with dilutions of 1 in $1,000,000$ and upwards the primary depressing action was absent. In experiments on other feebly beating hearts it was found that a dilution of 1 in 250,000 might not improve the beats, whereas a dilution of 1 in $1,000,000$ did. In general terms it may be stated that the more feeble the beats of 
the heart, the less is the dose of this drug required to induce a primary depression.

Each suprarenal of normal adult cats has, according to Elliott (14), an average adrenin content of about $0 \cdot 22 \mathrm{mg}$., so that, judged by these experiments, the sudden discharge of the whole of this into the animal's blood ought not to induce any decided primary cardiac depression. There is, however, the possibility that on the clinical side an endeavour to 'push' the drug might be followed by some such action. The existence of this primary depressing action indicates also a possible source of error in the use of adrenin to find the nature of the sympathetic supply to organs, for any action of the drug produced by large doses and not by small should ba regarded with some suspicion. The action of the large dose will, of course, be an action of adrenin, but not necessarily that of sympathetic nerves.

As already stated, the sympathomimetic action of adrenin gives a cardiac state in which the heart has an increased responsiveness to calcium. The development of this state under the influence of adrenin is antagonized by calcium, for, as shown elsewhere, calcium induces a cardiac state unfavourable to its other actions. Thus, a sufficiently small concentration of adrenin added to a perfusing solution containing a sufficient concentration of calcium may show no definite sympathomimetic action, whereas that same dose of adrenin added to a similar perfusing solution, but of much smaller calcium content, gives distinct sympathomimetic effects. Or, the experimenter may desensitize the heart to calcium by calcium, as shown elsewhere (7), and then use adrenin to overeome the effects already produced by the calcium.

Antagonisms between calcium salts and certain actions of adrenin have already been shown by Schrank (24) and Frankl (15). In each of these instances a simple antagonism was found, but from the experiments above it will have been gathered that the antagonism between adrenin and calcium salts is compound in the case of the heart, and we may not unreasonably suspect that the same is true for other tissues. We have to take into account at least four factors made up of the two types of actions of each substance. Each of them exerts a 'surface' type of action, i.e. one which comes and goes respectively with addition to or removal of the modifying substance from the perfusing solution. These 'surface' actions of the two substances are mutually antagonistic.

Each of them also has a 'deep' cardiac action revealing itself as a change persisting after removal from the perfusing solution of the substance originally producing it, and in this also the two substances are mutual antagonizers.

There are thus two pairs of mutually antagonistic factors which we may set out as made up of-

(a) The concentration of the adrenin.

$\left(a^{\prime}\right)$ The concentration in calcium of the perfusing solution.

(b) The state of the heart induced by the adrenin.

$\left(b^{\prime}\right)$ The state of the heart induced by the calcium.

The immediately apparent result in any particular experiment depends on 
which of these factors predominates. When the third factor predominates adrenin exercises a sympathomimetic action.

The understanding of these relations is quickly facilitated by a knowledge of the action of calcium in excitation processes. The bed-rock factor for consideration is the distinction that can be drawn between the working parts of the cardiac machinery and the accessories thereto (10). Excitation is a coagulative change evoked by calcium in certain colloids, and in the process we can distinguish two main factors, calcium tension and cardiac 'state' $(9,12)$, the latter being either electrical or physical, or both. Artificial or natural stimuli are to be regarded as agencies modifying this action of calcium. Bearing that in mind, there is no difficulty in visualizing the two mutually antagonistic actions of calcium itself, since we know that every efficient stimulus leaves behind it a refractory state.

The quicker an excitable organ overcomes or antagonizes the effects of a particular stimulus, the quicker does it become ready to respond again to a similar stimulus, so that adrenin, by antagonizing the refractory state produced by calcium, renders the heart also capable of responding more readily to the other actions of calcium. These antagonisms of 'state' between the two substances are those of chief interest from the physiological standpoint. The other antagonisms also have their interest, though they possibly play a relatively small part under normal conditions. The existence of these complex relations, however, gives scope for errors of interpretation in results. Those experimenters, for example, who only pushed the calcium, only found a single antagonism.

\section{The Antagonism between Adrenin and Potassium Salts.}

Frankl (15) found that adrenin was antagonized by potassium salts. The converse also appears to hold.

As is well known, the addition of potassium salts in moderate excess to a solution perfusing the heart is followed by a great slowing of the rate and general depression, ending finally in loss of electrical irritability.

The present experiments were performed in three main groups. In the one the calcium of the perfusing solution was present as the chloride and the chloride of potassinm was added to it. In the others the calcium was present as the dibasic phosphate, and the potassium was added to this, either as chloride or as dibasic phosphate also. The amount of potassium added was usually sufficient to stop the heart in the dilated and electrically inexcitable condition.

The addition of adrenin to these perfusing solutions was followed by a return of electrical irritability and a resumption of spontaneous activity. The amplitude of the spontaneous contractions returned towards the value existing. before the potassium salts were added to the perfusing solution, and might even surpass this if the amplitude on the original solution was distinctly less than the maximum possible for the heart. The return of excitability or the augmentation. 
of the amplitude of the spontaneous contraction in partially depressed hearts took place with great rapidity. A staircase action was observed, but the staircase was a steep one. At one moment the heart might be inexcitable to an induced shock, but two seconds later that same strength of stimulus elicited a good contraction.

On removing the adrenin from the perfusing solution, the heart continued to beat well for some little time longer, and then the condition of the heart appeared to be the same as that existing before the adrenin was added to the solution of extra potassium content.

If, however, the adrenin were not removed from the perfusing solution of the heart, it continued to beat in the presence of this extra potassium over long periods of time. The heart has been observed to continue beating with undiminished amplitude in presence of such amounts of potassium as were otherwise sufficient to stop it, for eight hours after the adrenin was added to the perfusing solution.

On removing the adrenin at the end of that time the heart reacted similarly to that observed when the adrenin solution had been perfused only a short time. The beats continued at the same amplitude for some time longer and then gradually diminished.

Some of the actions of adrenin are illustrated in Fig. 4 (A, B, and C).

In the illustrations to that figure there are first shown the effects which followed the addition of an excess of potassium to an otherwise adequate perfusing solution. The contractions rapidly fell away until they became just perceptible. Adrenalin hydrochloride was then added to the solution, with the result that the beart recovered its contractility and beat stronger than before. The second illustration, $4 \mathrm{~B}$, shows the beats of the same heart nearly four hours after the addition of the adrenalin to the solution of increased potassium content. It will be observed that during this interval there had been no diminution at all in the amplitude of contraction. The adrenalin was now removed from the perfusing solution, with the result that shortly after there was again a rapid failure of the spontaneous contractions. But in this case it will be noted that the antagonizing action of the adrenalin outlasted its actual removal from the perfusing solution. That is to say, the antagonism depends on some cardiac state. After the failure following this removal of adrenalin from the perfusing solution it was again added thereto, with the result that the heart beat well again. It was beating equally well three hours later, when the experiment was discontinued.

The balance of the inorganic elements in the solution just considered was such as to render it in every sense of the word an unsuitable perfusing solution. The addition of the adrenalin to it altered matters entirely. One had now a good perfusing solution. When experimentation on that heart was concluded, perfusion had been in progress some nine hours. During that interval no food material of any kind was supplied. Yet there had been practically no diminution in cardiac activity after that lapse of time. 
The perfusing solution considered above, regarded from the balance of its inorganic constituents, was unsuitable because of an excess of potassium. 'Unsuitable' perfusing solutions can also be constructed which owe their unsuitability to deficiency of one of the usual constituents. Be it understood that the term 'unsuitable' is employed here in a purely conventional sense as implying a mixture of inorganic salts on which hearts do not exhibit spontaneous activity for what is, again conventionally, a reasonable length of time. No rigid definition of these terms can be given, but their general sense will be understood from the remarks made above and below.

As an example of an 'unsuitable' perfusing solution, a Ringer with a calcium content represented by one-fifth saturation with the dibasic phosphate of calcium (= about 0.002 per cent. $\mathrm{CaCl}_{2}-\mathrm{Ringer's} \mathrm{solution}$ is usually made up with about ten times this amount). This solution has been met with earlier in this paper, and, as a variation, we will take here the modification of its ability to maintain cardiac activity induced by epinine. Epinine is a synthetic drug produced by Messrs. Burroughs and Wellcome, and is closely allied chemically to adrenin. It has a sympathomimetic action like adrenin (2) (Fig. 5).

The defects of the solution already considered above were chiefly due to the potassium being in excess; the defects of the solution now under consideration are chiefly due to a relative preponderance of the influence of the sodium over the calcium. The evidence for this statement will not be given here, the question having been in great measure dealt with by me elsewhere (7).

The beats of a heart which had been perfused for $1 \frac{1}{2}$ hours with this solution are represented on the extreme left of Fig. $5 \mathrm{~A}$, and are there shown to be very feeble. On addition of epinine to the solution the beats greatly increased in amplitude, and on its removal they fell away again. Repetition of the experiment gave the same result. The epinine was added again to the solution and the whole perfused continuously for one hour. It will be observed that at the end of that interval the amplitude of the beats was undiminished (extreme left of Fig. 5 B).

The epinine was then removed from the perfusing solution, with the result that the beats fell away, to be restored again when the epinine was re-introduced. A second removal of the epinine was again performed, with the result that failure again began. But in this case the failure was interrupted by increasing the calcium content of the solution to full saturation with the dibasic phosphate. The great and rapid increase of contraction which thereupon took place was sufficient to show that all that was required to enable the heart to exhibit good activity in a purely saline medium was the addition of more calcium to the perfusing solution. Reduction of the calcium content to one-fifth saturation was followed by a rapid failure, which was overcome by the addition of epinine once more. Three hours later the amplitude of the beats was still maintained, when the experiment was discontinued. Incidentally, it is, perhaps, of some interest to compare the 'curve of failure' which ensued on removal of epinine 
from the solution, and the diminution in calcium content. Like adrenin, epinine favours cardiac activity by inducing 'deep' changes, whereas the favourable action of calcium is a 'surface' effect. The 'curve of failure' following removal of epinine is concave to the base line, whereas that following removal of calcium is converse.

'Unsuitable' perfusing solutions were also constructed which owed their unsuitability to an excessive calcium content $\left(0 \cdot 05\right.$ per cent. to $0 \cdot 2$ per cent. $\left.\mathrm{CaCl}_{2}\right)$. Here again adrenin made possible a great prolongation of activity.

Thus, taking as a test of the suitability of a perfusing solution the fact whether the heart ean perform a satisfactory amount of work on that solution or not, we see that in presence of a trace of adrenin the 'balance' of the inorganic constituents becomes of secondary importance. In presence of a trace of adrenin a badly 'balanced' solution becomes more efficient to maintain cardiac activity than is a 'well-balanced' solution without adrenin.

\section{Some Differences between Blood and Saline Perfusing Solutions.}

Subsequent to the original work of Ringer one of the objects of those who devised inorganic saline perfusing solutions was to obtain one which in its 'balance' between the different constituents should approximate to that of blood. It is reasonable to believe that the balance in blood approaches perfection, but, owing to the different proportions between 'sorption ' and solution of the different salts, analyses of blood ash give little information as to this balance. Of one particular salt, it may be that one part is in solution to three parts associated with colloils; of another salt, the contrary relation may hold. The result is that the only real test one can apply is that of comparing the results of cardiac activity on solutions of different composition. It is so obvious that the longer a heart can maintain activity on a particular solution the better is that solution suited to cardiac activity, that there is apparently nothing to gainsay about it.

Yet it is possible to acquire some scepticism about these views by considering Addison's disease. The symptoms of the disease originally described by Addison are now known to be almost identical with those arising after excision of the suprarenal bodies. But, while agreeing that absence of the internal secretion of those bodies is a necessary antecedent to the actual appearance of the symptoms of this disease, it should at the same time be realized that the causa causans of those symptoms is to be sought in some peculiarity of the bodily mechanism. For some reason or other this mechanism minus the adrenal secretion is defective, and the symptoms of Addison's disease give us some measure of its defects. Inasmuch as the adrenal secretion is poured into the blood, it is possible that peculiarities in the composition of the latter may account for some, if not all, of the symptoms of Addison's disease.

That is the view which arises, I think, from the experiments below. The preliminary proposition to be put forward is that Ringer's solution never bas the balance of blood. 
This view is based on certain differences of reaction which are always obtained when the base of the ventricle is faradized immediately before and immediately after blood is substituted by an inorganic saline perfusing fluid. The strength of current used is the strongest that can be employed in the frog's heart perfused in situ; viz. one that caused feeble fibrillary twitchings of the pectoral muscles by spread, but without inducing any general contraction. If the strength of current be increased beyond this, the contractions of these muscles influence also the movements of the heart-recording lever.

Certain other precautions were taken. Thus, between the death of the animal and the procedure of replacing the blood in its heart by saline, there is the interval involved in the operations of inserting the cannula, fixing the heart to the recording lever, \&c. To eliminate any possible error arising from this source, a number of experiments were performed on spinal frogs in which observations were made at intervals from two or three minutes to one hour after destruction of the cranial contents. No qualitative change took place.

By using a strength of current sufficient to cause fibrillations of the pectoral muscles when the electrodes were touching the base of the ventricle, the local cardiac nervous mechanism was also brought under its influence.

On application of the faradizing current to the base of the ventricle under these conditions, there was usually evoked a ventricular contraction of about the same amplitude, or slightly greater than that of the previous spontaneous contractions. This was quickly succeeded by a second smaller contraction and a third one barely perceptible. Thereafter the faradizing current evoked at irregular intervals contractions of irregular amplitude, but smaller in amplitude than those existing before faradizing. Sometimes beats regularly alternating in strength were present, at other times the heart remained quiescent.

No two hearts ever reacted similarly. The description above merely gives some of the outstanding features observed. There was an apparent anarchy in the results obtained.

The explanation of the anarchy is simple. The faradization excited both the ventricle and the local nervous mechanism, with the result that it became subject to mixed forces of inhibition and excitation. Inhibition always predominated in the blood-containing heart, and the anarchy of results was due to irregular partial breaks in this inhibition.

Directly after the blood in the heart had been replaced by any of the usual inorganic saline perfusing solutions, the reaction of the heart to the same faradizing current was radically changed. Inhibition disappeared, or at any rate gave no definite evidence of its existence. Instead the heart reacted to the faradizing current in a manner consonant with reasoning employed by Engelmann, viz. that by using a rapid faradizing current some one of the shocks will fall on the heart during diastole approximately at the point where the returning excitability associated with diastole renders the heart excitable to the strength of shock used. The contractions now evoked by the artificial stimulus came on so early in diastole that the heart was unable to relax completely. The 
contractions evoked by the artificial stimulus also started from a different base line, the summation line, than that from which the spontaneous contractions started. These artificially evoked contractions came on so much faster than before and frequently succeeded each other so quickly as to give almost complete fusion. Under certain conditions a condition resembling the tetanus of skeletal muscle could be obtained. An example of the change taking place in the reaction of the heart to faradization on passing from blood to inorganic saline perfusing solution is given in Figs. $6 \mathrm{~A}$ and $6 \mathrm{~B}$.

The type of change shown in the diagram was constantly obtained on passing from blood to an inorganic saline perfusing solution. Several hundred experiments were made, and the perfusing solutions comprised all those recommended as suitable for the heart, and many others as well. Provided the saline solution was one that enabled cardiac activity to be maintained for half an hour, it was also one incapable of allowing the heart to show those predominantly inhibitory phenomena seen immediately provious to its perfusion. No matter how well a given inorganic saline mixture enabled a heart to maintain spontaneous activity, there was always that difference observed when it was substituted for blood as a perfusing solution. There is something in the composition of these solutions which renders them vastly different from blood.

The immediate impression arising from these results is that inorganic saline perfusing solutions destroy the local inhibitory mechanism of the heart. That is not so, however, for predominantly inhibitory effects produced under the circumstances mentioned above in hearts perfused with inorganic saline mixtures after they had been treated with high concentrations of potassium salts (1-5 per cent.). The return of inhibition under such circumstances was temporary and corresponded to the period of ' loading' of the heart with potassium (5). But it was sufficient to show that these perfusing solutions were unsuitable media for the manifestation of inhibition, rather than destroyers of the local inhibitory mechanism. An example of such 'return' is shown in Figs. 7 A-7 E.

Two other points about this change, and, I think, indicative of its nature, are: (1) It took place immediately after the salt solution was substituted for blood. (2) The summation line was raised.

The former places the phenomenon in that group of actions I have termed surface actions (7). Experiments performed in other directions show that a change in the relative proportions of the inorganic salts present would produce this. A change of state would probably take longer than the time interval elapsing in these experiments. A change of state can be finally eliminated by the facts that the blood-containing heart is highly sensitive to calcium and gradually loses this sensitiveness when perfused. The change actually shown is, thus, opposite to that shown by the heart on the assumption that the phenomena now considered were due to a change of 'state'.

A raising of the summation line is always produced in the perfused heart when either the response of the heart itself to calcium or the tension of the 
calcium in the perfusing solution is raised. But having already eliminated changes in 'state', we are left with an increased tension of calcium as responsible for the change. The validity of the premises leading to this conclusion is enhanced by the fact that, using entirely different methods, Keith Lucas (19) has already demonstrated that Ringer's solution has a greater calcium tension than blood.

The changes taking place in the level of the summation line when the tension of calcium in a saline perfusing solution is increased are well shown in Plate 25, Figs. $8 \mathrm{~A}$ and $8 \mathrm{~B}$.

This greater tension of calcium may either have been due to the perfusing solution containing an absolutely greater amount of calcium than blood or a relatively less amount of sodium or potassium.

We may at once deal with the sodium. The perfusing solutions contained 0.6 per cent. sodium chloride, which is isotonic with the red blood corpuscles of the frog. As shown by my former experiments, a diminution in the amount of sodium chloride would have led to a raising of the summation line immediately after the one solution was substituted for the other (7), (8). To attempt to account for the raising of the summation line in terms of a smaller content of these perfusing solutions in sodium chloride was thus impossible, because it meant the assumption that blood contained a greater tension of sodium chloride than that which is isotonic with it.

There was a difference in the reaction of the two solutions, the saline solution being more alkaline than blood. The difference was such as according to my previous experiments would raise the summation line, but not within the limits of time here considered. On the contrary, the effects observed should have been those of depression (8).

A series of experiments was then made in which the changes were observed on passing from blood to a series of solutions constant in composition except as regards their calcium content, each experiment being made on a separate heart. The basic solution contained 0.6 per cent. sodium chloride; 0.03 per cent. potassium chloride; 0.01 per cent. sodium bicarbonate.

It was soon found that no mere reduction of calcium content of these solutions could make the summation line of the fresh heart coincide with the base line of the spontaneous contractions and leave the heart capable of maintaining functional activity. A point was always reached where the amplitude of contraction on passing from blood to perfusing solution made a sudden drop and then more slowly increased again towards its former value.

The reason of these variations in the amplitude of the spontaneous contractions is again made clear by former experiments. They were such as take place when the balance between sodium and calcium is disturbed in such a way as leaves the former preponderating over the latter (7).

The final element to be considered was potassium, and this apparently afforded the key to the problem. Phenomena of inhibition under the conditions mentioned above returned- 
(a) After hearts had been treated with large doses of potassium salts.

(b) During the period of failure associated with the perfusion of solutions containing sufficient potassium to stop the heart in the dilated condition, or at any rate to depress greatly its activity.

The return of inhibition under the former condition was only temporary. But it was the only way in which I found it possible to obtain decided inhibitory phenomena by the method used in presence of a solution of inorganic salts alone capable of maintaining good cardiac activity. The dose of potassium had to be a large one (above 1 per cent.), and the inhibition was obtained at a time when, according to the evidence in a previous paper, the tissues could be considered as containing large amounts of potassium. A relative excess of potassium in the tissues thus seemed to be a factor favouring inhibition. This accords with the work of Bottazzi (3), Howell (16), Macdonald (20), and others.

But on attacking the problem the reverse way, namely, by using solutions containing more and more potassium, it was found that as the potassium content of the solutions was increased, so the phenomena obtained by the experimental method used tended to approximate nearer and nearer to those of inhibitory type. But they did not become definitely so until the amount of potassium added was such as markedly interfered with the activity of the beart.

The addition of adrenin to these solutions not only enabled the heart to maintain activity on them, but also to preserve some phenomena of inhibition. Thus, supposing the start was made with a saline solution on which the heart beat well, but on which it showed no evidence of inhibition under, the experimental conditions; instead faradization evoked excitatory activity with a summation line about two-thirds as high as the summits of the recorded spontaneous contractions. On adding potassium to such a solution sufficient to stop the heart in the dilated condition, faradization of the heart elicited inhibitory phenomena during the period of failure. Then, on adding adrenin to this new mixture, it was easy to get the heart to beat as well as it had done previously before the addition of potassium. But the summation line lagged behind. Whereas previously it was raised, it was usually now at the same level as the base line. After replacing this mixture by the original saline solution, the same jump in the summation line took place as was the case on passing from blood to saline mixtures.

It would have been of interest, perhaps, to have discovered a saline perfusing solution on which the heart behaved exactly as it does on blood. The war and other circumstances have, however, stopped experiments for the present.

If, however, we consider the points established above-

1. That the frog's heart shows a radical change in its behaviour immediately after Ringer's solution is substituted for blood;

2. That this change of behaviour, loss of predominance of inhibition, is not due to destruction of the local inhibitory apparatus, but to Ringer's solution being an unsuitable medium for its manifestation; 
3. That this unsuitability of Ringer's solution is due to its relatively greater calcium tension than blood;

4. That the relatively greater calcium tension of Ringer's solution is due largely to an absolutely diminished potassium tension;

5. That a loading of the heart with potassium temporarily brings back predominantly inhibitory phenomena;

6. That traces of adrenin overcome the inhibitory action of certain perfusing solutions containing an excess of potassium less than their action on amplitude of contraction;

we have, I think, a sufficient body of evidence to indicate the origin of certain symptoms of Addison's disease.

Much of the work done on the suprarenals, prior to that by Oliver and Schäfer (21), was directed towards showing that their secretion neutralized toxic bodies. The nature of these toxic bodies was unknown, but they induced definite symptoms such as muscular weakness, shown by Abelous and Langlois (1) to be localized to the nerve endings, cardiac feebleness, and low blood-pressure. The general tenor of these experiments and the views arising therefrom are well presented by Rolleston ( 23 ).

But with the discovery of adrenin there was discovered a positive entity producing definite physiological results and accordingly modifying views in regard to the action of the suprarenals.

The view arising from the present experiments is a sort of half-way house between these. Taking first of all the only possible test of the suitability of a perfusing solution, namely the relative capacity of the heart to maintain activity on it, we have seen that in presence of traces of adrenin the balance between the inorganic constituents becomes of secondary importance. Varying extremes of unsuitability due to lack of 'balance' between the inorganic constituents were taken, and it was shown that the addition of traces of adrenin to these solutions rendered them better than the best balanced inorganic salt solution in their capacity for maintaining cardiac activity. Comparing next certain activities of the blood-containing and the perfused heart, it was shown that the behaviour of the blood-containing heart never approximated to that of the heart perfused with a 'well-balanced' inorganic mixture. Approximation of behaviour only began when the inorganic mixture became overbalanced in a certain direction.

Now, we have every reason for believing that adrenin is a normal constituent of blood. We also know that certain defects arise with its absence from blood. From the experiments above we saw that in presence of traces of adrenin 'balance' between the inorganic constituents of a perfusing solution becomes of secondary importance. They indicated also that blood is an 'unbalanced' solution in which the action of potassium predominates. Of the action of such a solution we have some knowledge. It enfeebles cardiac action, decreases vascular tone, and renders muscle easily fatigued. We have not the same knowledge in regard to its action on the skin, gastro-intestinal system, \&c. But, where we have the knowledge, the action corresponds to the symptoms of Addison's disease. 


\section{Concluding Remarks.}

The material of the paper will have been found to divide itself conveniently into three main parts. The first of these dealt with the relations between calcium and adrenin, and is the one to which further attention will now be given. The relations found were those of a mutual antagonism and reinforcement. They give a good illustration of the principle laid down before that the temperament of an organ depends on the calcium content of the solution perfusing it (10). In the present case we have a substance exerting a depressing and an augmenting action on the heart, each of which is antagonized by the calcium, so that whichever of these actions adrenin tends to exert at a given moment, its realization is rendered more difficult or more easy according as calcium is also present in greater or less amounts.

Regarding the results from a more general standpoint, we see in adrenin a device for enabling the organism to carry out its functions on a solution containing less amounts of calcium than would otherwise be necessary, and ipso facto endowing it with a greater power of reacting to its environment.

It is also of some interest to consider the relations between the augmentive and the depressive actions of adrenin. When small doses of the substance were employed the effects found were such as could be ascribed to sympathetic stimulation, whereas the large doses gave 'inhibition' and 'inhibitory rebound'. There was, however, no dividing line found between these two apparently diametrically opposite actions, but instead the one action gradually merged into the other. The possibility then arises that both these actions represent different aspects of certain fundamental effects of the drug. That such is the case is rendered possible by the grading effects shown in these experiments, and on the theoretical side they have a ready explanation in certain theories of excitation and inhibition already advanced $(10,11)$.

Modifying Macdonald's theory, I have suggested that in excitation there is a coagulative change in certain colloids through formation of a calcium compound (10). According to him this coagulative change is accompanied by a release into watery solution of previously absorbed potassium salts which now confer a positive charge on the colloids whence they came. This electric charge in its turn reverses the coagulative change in the colloids and so brings conditions back towards the original state of affairs. During inhibition Macdonald (20) considers that a maintained positive charge produces a finer state of colloidal subdivision than normal with a coincident withdrawal of more potassium salts from watery solution. I have shown, however, that a positive charge renders the cardiac colloids incapable of combining with calcium and can decalcify them as well as does an oxalate(6), and also that sodium may be a factor in determining a finer state of subdivision of the colloids (7). Modifying Macdonald's theory in accordance with this action of the positive charge, one sees as a prime factor in inhibition an inability on the part of the inhibited tissue to combine with calcium. The state of aggregation is of secondary importance, and 
is indeed one which greatly favours the action of calcium. Both views, however, present inhibition and diastole as resulting from the action of a single agency. Diastole is an inhibition of systole, and inhibition itself an exaggeration of the action producing diastole.

Now, we know that the heart is normally quickened at the expense of the diastolic period, and that adrenin is a substance which quickens the diastolic process. The inhibitory action of adrenin is explicable on the assumption that it depresses the capacity of calcium to combine with the cardiac colloids, and its sympathomimetic action is explicable on the assumption that it produces a state of the heart the opposite of the coagulative. If the excited and therefore nonexcitable heart be one in which there is a coagulative change resulting from the formation of a calcium-colloid compound, both these actions of adrenin should hasten its reversal. If, however, we consider the series of experiments performed on one heart, in which the degree of improvement following exposure to successively decreasing doses of adrenalin was noted, and draw a curve by plotting improvement against concentration of adrenalin, the curve becomes flattened after concentrations of one part in ten millions. That concentration presumably represents what was the maximum dose giving improvement in that heart consistent with economy of drug. It may be regarded as the counterpart of an oxygen tension of $150 \mathrm{~mm}$. $\mathrm{Hg}$ in its relation to blood. At that tension blood is practically saturated with oxygen. It can take up more oxygen if the oxygen tension be increased further, but the amount so taken up is now very small relative to any such increase. Similarly the drug at the concentration mentioned had nearly reached its maximum as regards producing an excitable state. Pushing the drug further had little further action in this direction. Its decalcifying action did not, however, have a similar limit, so that with this further pushing of the drug it tended to predominate. When it was paramount, a heart was obtained in a highly excitable 'state', but incapable of being excited on account of its incapacity to combine with calcium. On removing the adrenin, the agency rendering the heart incapable of combining with calcium was also removed, but not the 'state' of the heart induced by the same agency. The latter took some further time to subside, and during that time we bad the ' inbibitory rebound ' to augmented activity which was found to be similar to the visible sympathomimetic action of the drug.

Thus, adrenin appears as a substance exerting two fundamental actions on the heart, each of which has a different maximum. The visible effects of these actions depend on the adjustment between them. I would not claim that the adjustments I have obtained between them are other than coarse. The body probably attains a finer adjustment and with it further refinements of its action, and so produces augmentive, accelerator, or initiative effects as they are necessary. There may be in the sympathetic nerve fibres mediating each of these effects, but from what has been stated above we see the possibility of such effects resulting from a grading of the adjustment between two fundamental actions of these nerves. 


\section{Addendum on Epinine.}

Epinine is a substance prepared by Messrs. Burroughs \& Wellcome. It is closely allied to adrenin, differing from the latter only in the absence of the alcoholic hydroxyl group in the side chain. First synthesized by Pyman (22), its physiological action was shown by Barger and Dale(3) to approximate very closely to that of adrenin.

In regard to its sympathomimetic action, all that has been said above holds good for epinine, one of the illustrations there concerning perfusing solutions showing this action of epinine. The action of this substance, thus, bears out what has already been said in this paper.

Like adrenin it can also exert a depressing action on the heart when used in large doses. This depressing action, however, is unlike anything else $I$ have met. Thus, I used a strength of epinine of one in twenty-five thousand and perfused it in a Ringer solution one-fifth saturated with calcium phosphate. The heart began to fail though rather slowly. Before failure had gone far the epinine was removed from the solution, but the fuilure went on in spite of the removal. It was traced as continuing progressively during a further period of ten minutes by means of solutions of increasing calcium content. After the failure on the solution mentioned above, the heart beat well on a solution fully saturated with the calcium phosphate, but then failed again. A Ringer containing 0.025 per cent. $\mathrm{Ca} \mathrm{Cl}_{2}$ was next employed and with a similar result. After that a solution containing 0.15 per cent. $\mathrm{Ca} \mathrm{Cl}_{2}$ was used, also with a similar result. With each new solution of higher calcium content than that previously used there was a fresh outburst of activity followed by a failure.

At the end of that series the contractility of the heart as estimated by potassium chloride was found undiminished. But on evoking a series of such contractions it was next found that these also diminished in amplitude in succession. In some cases potassium salts ceased eventually to produce contraction.

The action was, however, in some measure reversible because with continued perfusion of the Ringer's solution contractility to potassium chloride and excitability to induced shocks began to return. After 1 in 5,000 epinine at least one hour elapsed between the original failure and the subsequent visible return of spontaneous contractility. But on the latter solution a 50 per cent. return only was obtained.

In other experiments the failure was not carried out to the extent mentioned above. The process could not be rigidly controlled, but it was found possible to render the heart incapable of beating well on a particular solntion, but capable of beating well on a similar solution but of higher calcium content. Thus, a heart beating well on a Ringer solution containing 0.05 per cent. calcium chloride ceased spontaneous activity when 1 in 250,000 of epinine was added thereto, and did not resume activity when the epinine alone was removed. But 
it immediately resumed activity when the calcium content of the solution was increased to $0 \cdot 025$ per cent.

The conclusion established from these experiments, that epinine used in the strength and under the circumstances mentioned above desensitizes the heart to calcium, was confirmed by using adrenin. Hearts perfused with a particular solution and rendered completely inexcitable to induced shocks as a result of treating them with epinine, could bave that excitability restored by appropriate addition of adrenin to the same solution.

Now, if any particular change induced by epinine was due primarily to chemical changes we ought to enhance that change by increasing the epinine concentration. The evidence, such as it is, indicates that the 'epinine-heart' compound is unsuitable to show the normal activity. If, however, we take the view that the state of aggregation of certain cardiac colloids is a factor determining the outward manifestations of its activity, a physical change induced by a given drug can be regarded as sufficient to influence such activity. The indication is that sympathomimetic actions are primarily the result of physical rather than chemical changes.

The experiments are also of interest in demonstrating the delicate adjustment of the animal organism to its own drugs. Epinine and adrenin have similar physiological effects, but when we employ them in what may be considered as pathological concentration we get evidence of a certain lack of adaptation of the tissues to an environment containing epinine. It is conceivable that causes originally leading to the animal synthesis of adrenin produced also allied bodies, but evolution is behind the present adaptation to adrenin, and it seems possible that it represents a survival of the fittest.

\section{Summary.}

1. Adrenin has a twofold action on the frog's heart, a primary depressing action resembling inhibition, and an augmenting action, the sympathomimetic action.

2. Three factors are found to be concerned in the depressing action of adrenin.

3. The relations between adrenin and calcium are found to be fourfold.

4. The evidence is given that traces of adrenin render 'balance' between the constituents of an inorganic saline perfusing solution of secondary importance in regard to their suitability as media for the manifestation of cardiac activity.

5. It is shown that the behaviour of a heart perfused with a 'well-balanced' Ringer's solution never approximates to the behaviour of the blood-containing heart and that the change takes place immediately after the one solution replaces the other.

6. It is shown that as inorganic perfusing solutions become unbalanced in a certain direction, so the behaviour of the heart on them tends to approximate to that of the blood-containing heart. 
7. Such solutions contained amounts of potassium sufficient to interfere with cardiac activity.

8. Traces of adrenin rendered them capable of maintaining cardiac activity and of preserving in great measure the resemblances between the behaviour of the heart perfused with them and of the heart containing blood.

9. On the basis of these experiments it is suggested that the proportions between the inorganic constituents of blood are such as to render it an unsuitable medium for the manifestation of cardiac activity except in presence of adrenin. The origin of certain symptoms of Addison's disease is traced to such lack of balance.

\section{REFERENCES.}

1. Abelous and Langlois, Arch. de Physiol. Norm., Paris, 1892, xxiv. 269. 465.

2. Barger and Dale, Joum. of Physiol., Camb., 1910-11, xli. 19.

3. Bottazzi, Arch. de Physiol. Norm., Paris, 1896, xxviii. 882.

4. Brodie and Dixon, Journ. of Physiol., Camb., 1904, xxx. 476.

5. Burridge, Quart. Journ. Exper. Physiol., Lond., 1912, v. 347.

6. Burridge, ibid., 1914, vii. 167.

7. Burridge, ibid., 1915, viii. 303.

8. Burridge, ibid., 331 .

9. Burridge, Quart. Journ. Med., Oxford, 1915-16, ix. 43.

10. Burridge, ibid., 271.

11. Burridge, ibid., 1917, x. 157.

12. Burridge, Jowrn. of Physiol., Camb., 1914-15, xlix (Proc. Physiol. Soc.), p. xlii.

13. Elliott, ibid., 1905, x xxii. 401.

14. Elliott, ibid., 1912, xliv. 374.

15. Frankl, Pfingers Arch.f. d. ges. Physiol., Bonn, 1909, exxx. 346.

16. Howell, Amer. Jourm. of Physiol., 1905-6, xv. 280.

17. Howell and Duke, Jourm. of Physiol., Camb., 1906-7, xxxv, 181.

18. Langley, ibid., 1901-2, xxvii. 237.

19. Lucas, ibid., 1908, xxxvii. 459.

20. Macdonald, Proc. Roy. Soc., Lond., 1905, lxxvi. B. 322.

21. Oliver and Schäfer, Journ. of Physiol., Camb., 1895, xviii. 230.

22. Pyman, Journ. Chem. Soc. Trans., 1910, xcvii. 264.

23. Rolleston, Goulstonian Jectures on Suprarenal Bodies, Lond., 1895.

24. Schrank, Zeitschr. für klin. Med., Berlin, lxvii. 230. 


\section{DESCRIPTION OF FIGURES.}

Puate 21, Fic. 1. Heart faradized between the signs + and $\uparrow$.

At $\rightarrow 1 / 20,000$ Adr. one part in 20,000 of the Parke Davis preparation perfused.

This was perfused at high pressure to overcome the resistance of the partially contracted heart. The first sudden rise of the summation line is due to this sudden rise of perfusion pressure (Pr.).

At $P$ o the perfusion pressure was reduced to normal and summation line fell slightly in consequence.

FIG. 2. From the first arrow on the left to the extreme right the heart was subjected to solutions containing 1 in 25,000 adrenalin hydrochloride.

At $\uparrow \frac{1}{5}$ the calcium content of the solution was that of one-fifth saturation with the dibasic phosphate.

At $\uparrow 1$ the calcium content was that of full saturation.

Here and elsewhere CaHP signifies a perfusing solution containing 0.6 per cent. $\mathrm{NaCl}$, $0.03 \mathrm{KCl}$ and saturated with the dibasic phosphate of calcium. A fraction sign in front, e.g. $\frac{1}{5}$ CaHP, indicates a similar solution, but with its calcium content reduced according to the fraction.

Plate 22, Fig. 3. On the extreme left the heart was beating on a solution fully saturated with the dibasic phosphate of calcium.

At $\rightarrow \frac{1}{5}$ CaHP the calcium content was reduced to one-fifth saturation.

At +1 in 250,000 that strength of adrenalin hydrochloride was added to above.

$\rightarrow \frac{1}{5} \mathrm{CaHP}=$ that solution without adrenalin perfused.

FrG. 4 A. On extreme left heart was beating on a solution saturated with the dibasic phosphate of calcium. The failure there seen is due to the addition of 0.05 per cent. of the dibasic phosphate of potassium $\left(+0.05 \mathrm{~K}_{2} \mathrm{P}\right)$.

At +1 in 1,000,000 Adr. perfusing solution made up to that strength in adrenalin hydrochloride.

1.15 represents time of day.

FIG. 4 B. Beats some four hours after A.

$\rightarrow$ Adr. off. Adrenalin removed from the perfusing solution.

$\longrightarrow+1$ in $1,000,000 \mathrm{Adr}$. Adrenalin replaced.

Note that although four hours had elapsed between taking the tracings of beats on the extreme right of both figures there has been no measurable reduction in amplitude. Perfused with a 'well-balanced' Ringer alone, the frog's heart would show a 25-50 per cent. reduction in a similar time.

Plate 23, Fig. 5 A. The feeble beats seen on the extreme left represent what would be 'normal' amplitude for a heart perfused with the 1 CaHP solution after the time which had here elapsed since perfusion had been begun. The waxing and wanings seen represent the effects following addition of epinine to that solution and its subsequent removal. The 'breaks' in the tracings taken on the slow-moving drum are artificial, and are usually the result of floor vibrations.

Frg. 5 B. The epinine introduced at 3.50 p.m. (see $5 \mathrm{~A}$ ) was removed at 4.50. There was, bowever, no measurable diminution in amplitude during that interval. The remainder of the tracing is devoted to showing -

(1) That epinine was necessary for activity on the $\frac{1}{5}$ CaHP solution ;

(2) That the beats were feeble on the 1 CaHP solution, because of its small calcium content-perfusion of CaHP solution.

See also remarks in text.

FIG. 6 A. The base of the ventricle was faradized between the marks + and $\uparrow$.

Up to the white square the heart contained blood. At the white square the blood was expelled from the heart and replaced by a saline perfusing solution. The irregularities $P . V$. are the result of manipulation.

Immediately after replacing blood by the saline perfusing medium faradism evokes a condition resembling tetanus.

Note.-The perfusing solution used here was specially selected as giving an exaggeration of the effects usually seen, since here the change is from inhibition to tetanus approximately. The reaction on the usual Ringer is shown in Figs. 1, 7, 8. 
FIG. 6 B. Another example of faradism of blood-containing heart. Fast-moving drum on extreme left.

Plate 24, Fig. 7 A. On the extreme left the change had just been made from blood to Ringer. The summation-line is now about two-thirds the height of the apices of the spontaneous contractions as recolded.

Heart then treated with potassium chloride- 5 per cent. $\mathrm{KCl}$.

At $N . S$. potassium solution replaced by 'Ringer'.

$P . R$. Perfusion pressure suddenly raised (vide Burridge (5)).

$P . V$. Notches in tracing are artificial here and due to pumping saline through heart.

It will be noted that immediately after spontaneous beating had been resumed complete inhibition was obtained on faradizing the heart.

FIG. $7 \mathrm{~B}$ is a direct continuation of $7 \mathrm{~A}$.

At 0.15 per cent. $\mathrm{CaCl}_{2}$ the calcium content of the solution was increased to that amount with the result that excitation gradually predominated over inhibition until a condition resembling tetanus was produced.

FIG. 7 c. Shows effects following faradism before treatment with potassium chloride.

FIG. $7 \mathrm{D}$ and E. Shows change in reaction to faradism after treatment with potassium chloride. Fig. $7 \mathrm{E}$ is a direct continuation of $7 \mathrm{D}$.

Plate 25, Fig. 8A. The heart was alternately perfused with a Ringer containing 0.02 per cent. and 0.15 per cent. of $\mathrm{CaCl}_{2}$ respectively.

The changes seen here should be compared with the changes seen in Fige. $6 \mathrm{~A}$ and $6 \mathrm{~B}$.

Heart faradized between marks + and $\uparrow$.

Fra. 8 B. Faradization was begun at a point anterior to extreme left of tracing and continued up to the point marked ' $\uparrow$ 'off'.

The fluctuations in the level of the summation line following changes in calcium concentration of the perfusing solution are here shown. 


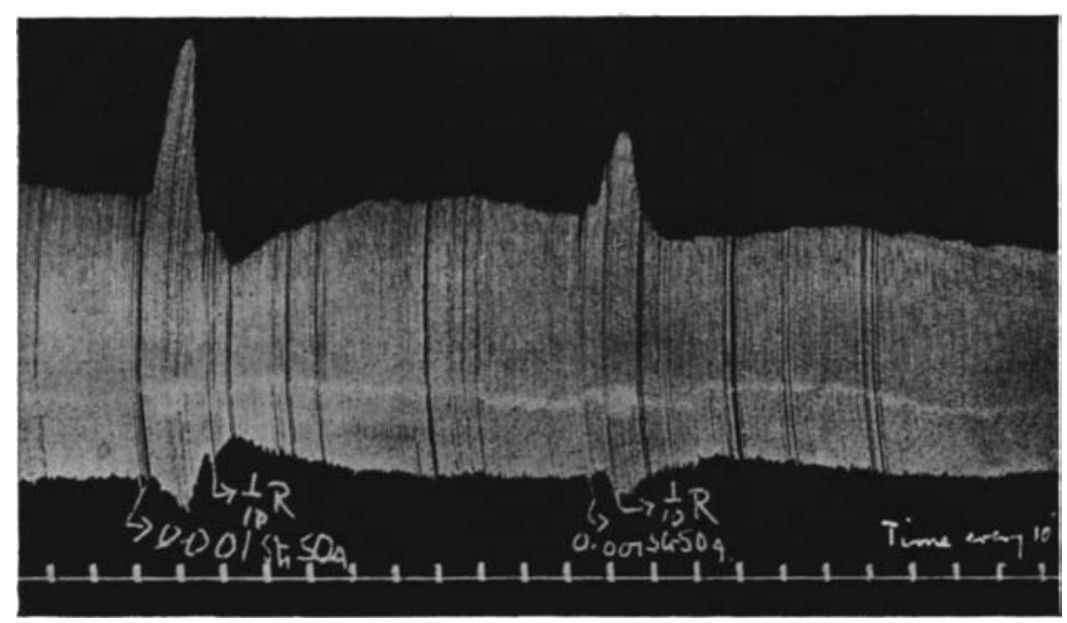

Strychnine

FIG 1

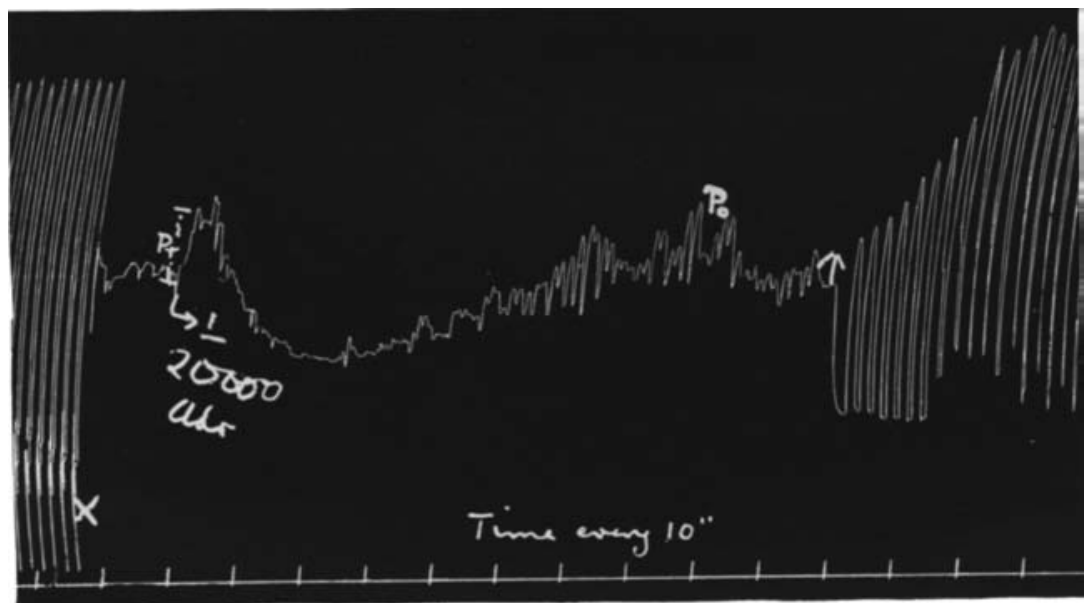

Adrenin

FIG. 1

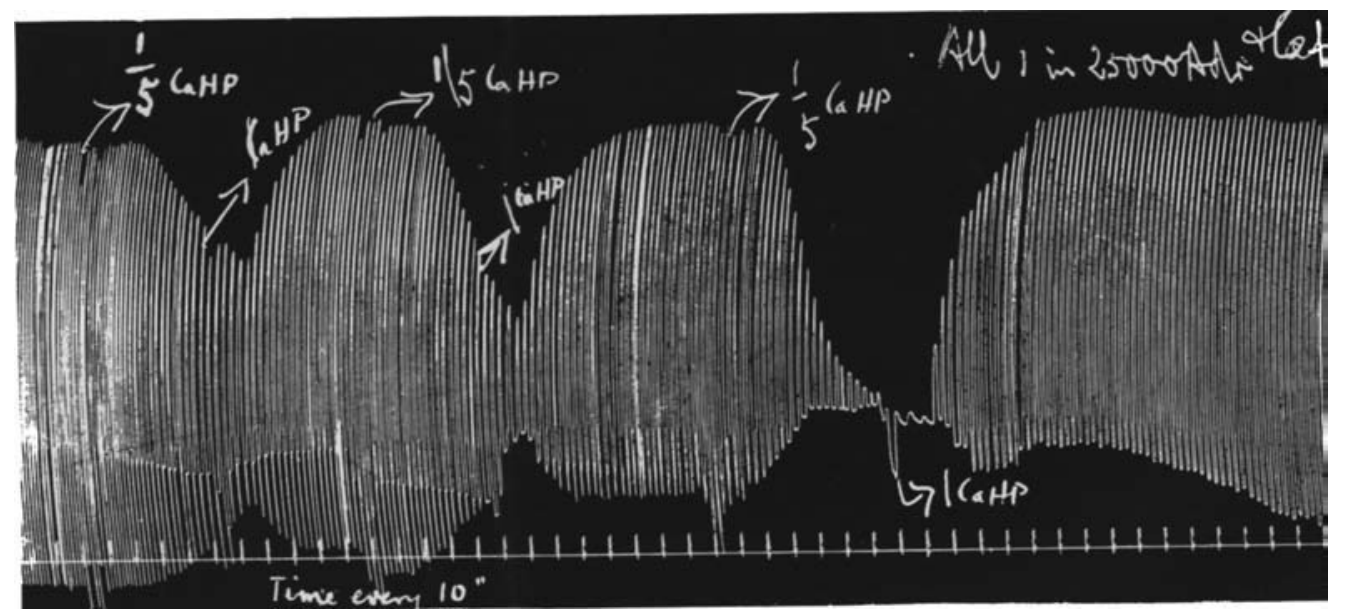

FIG. 2 

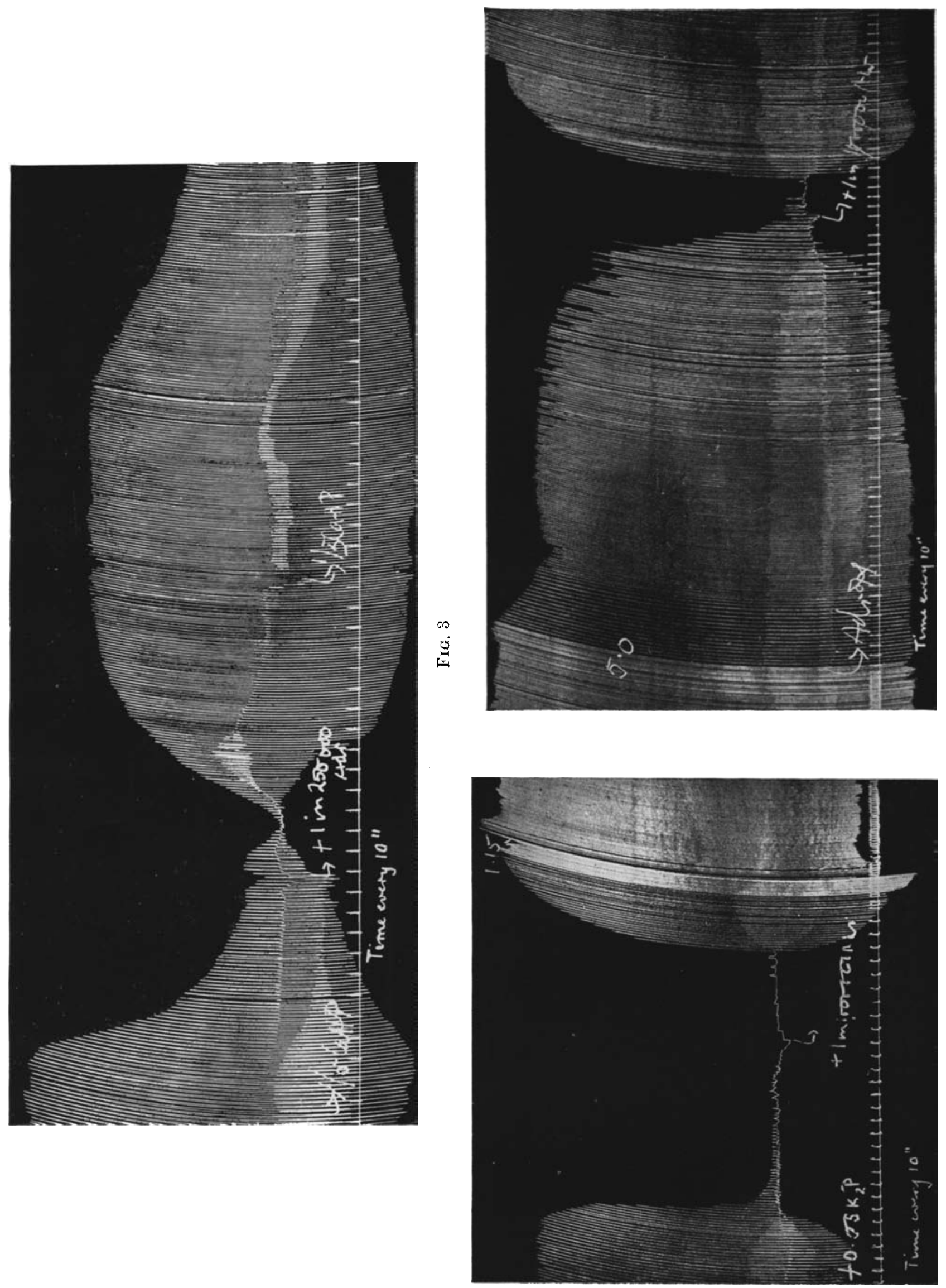


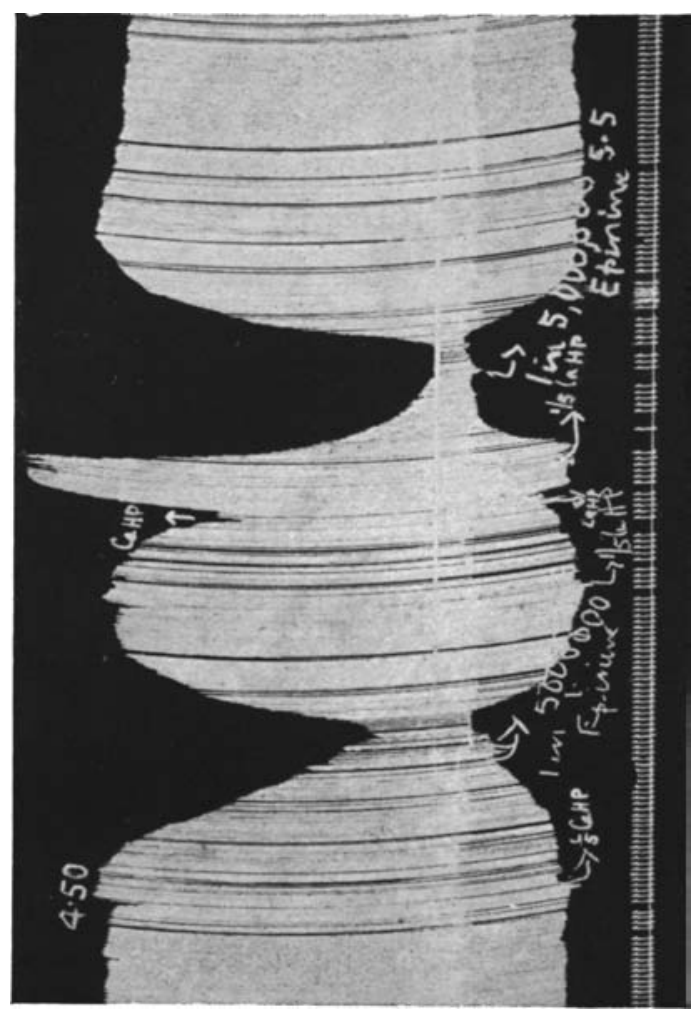

$\varphi$
20
0
0
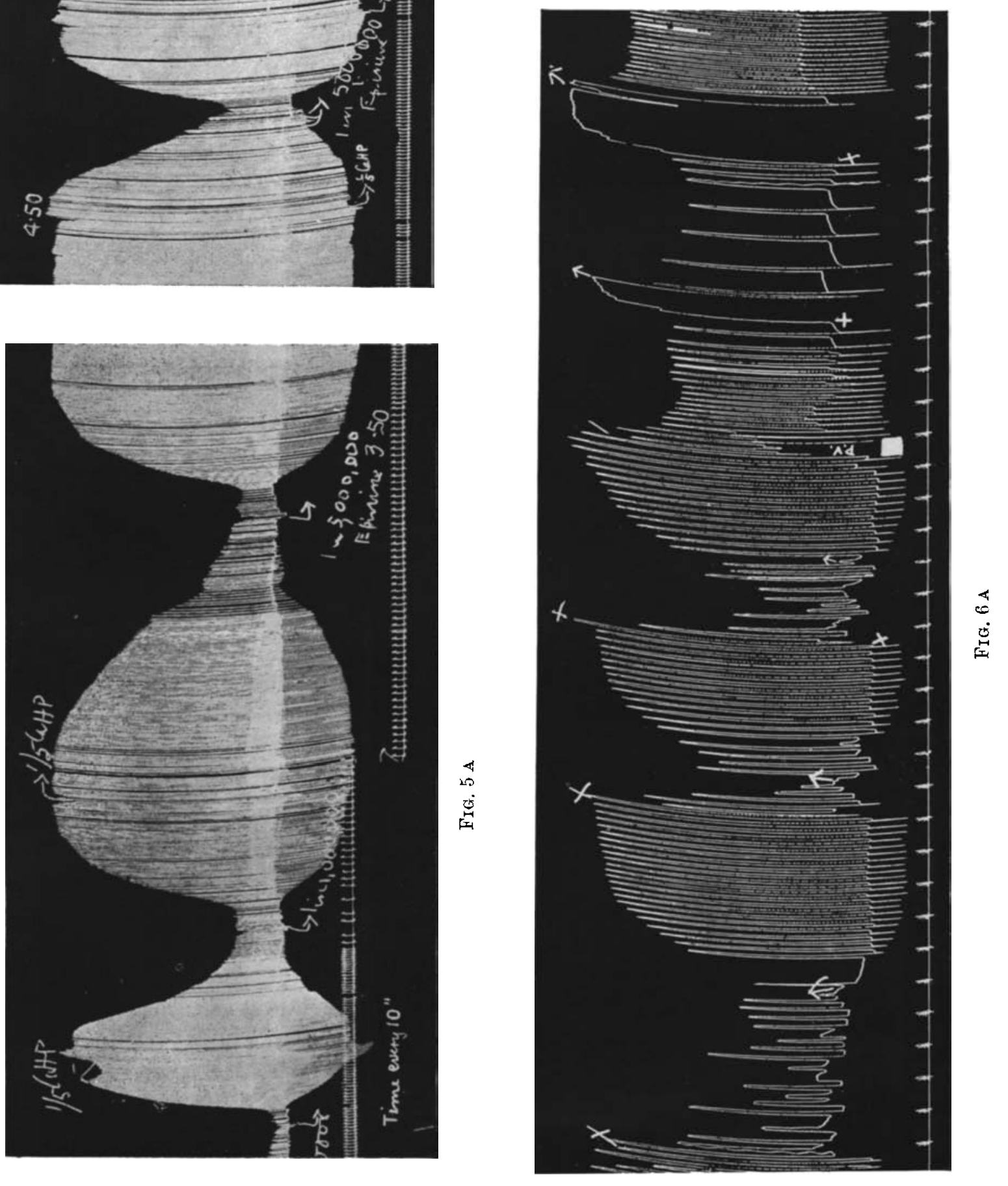


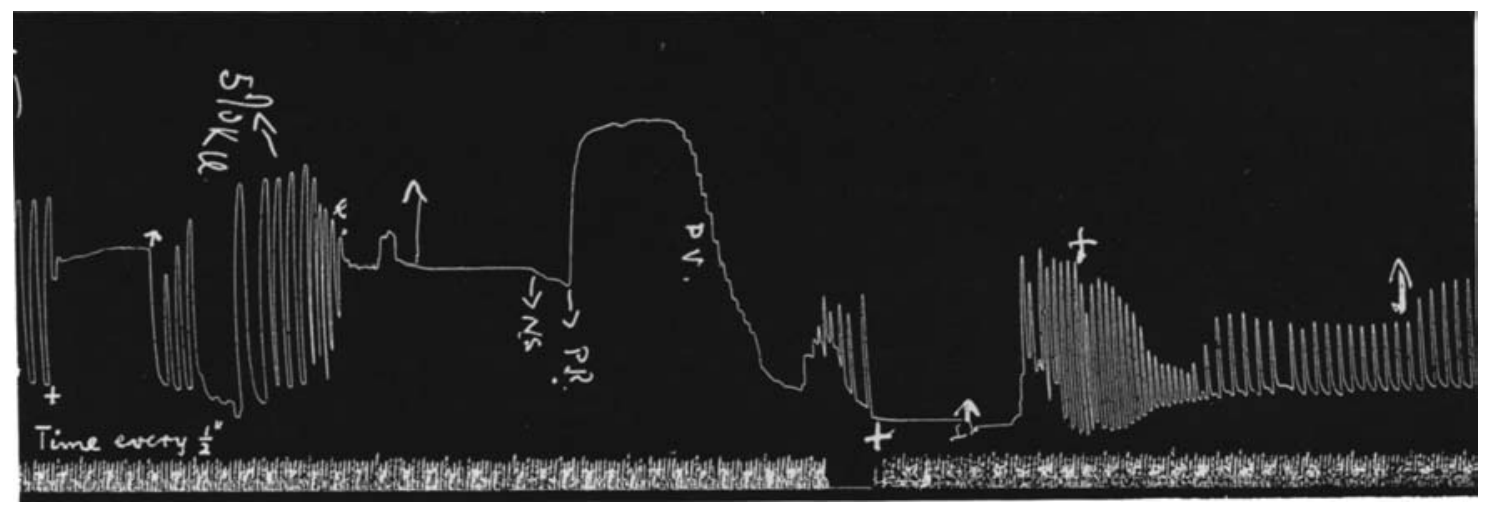

FIg. $7 \mathrm{~A}$

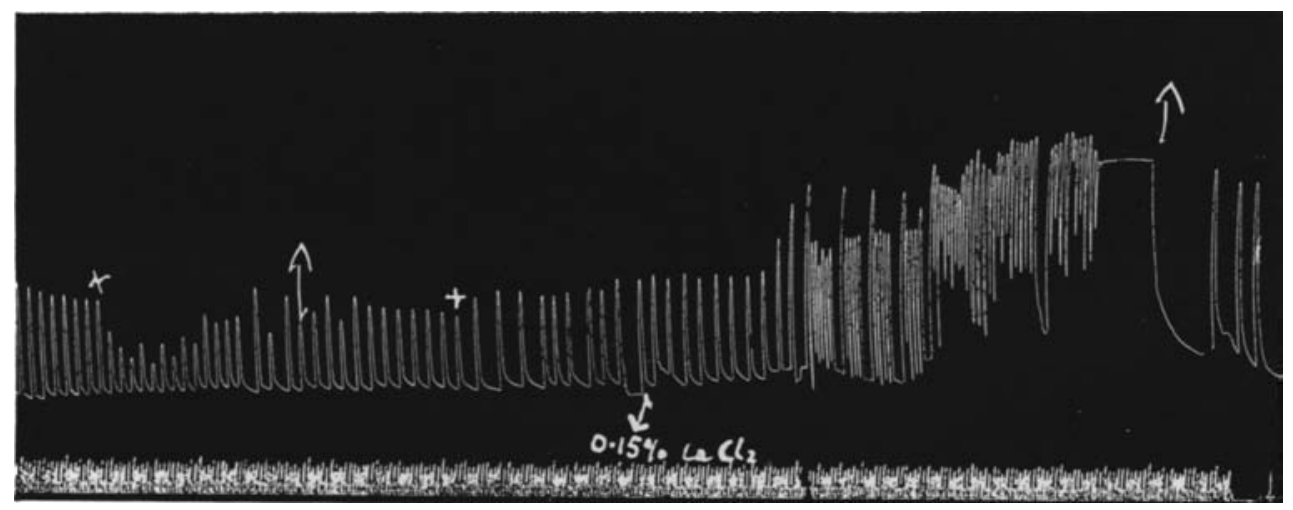

FI

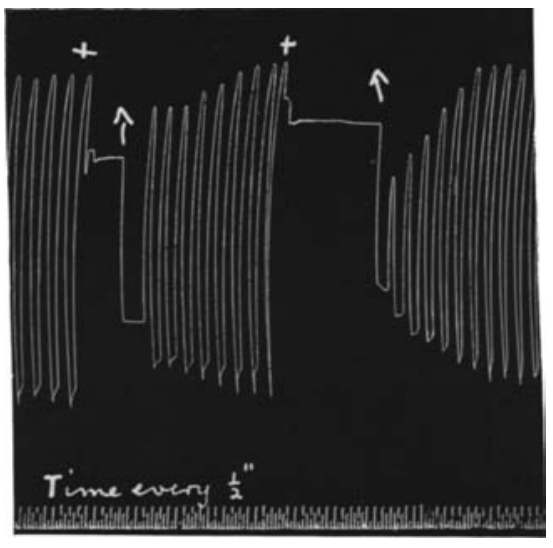

FIG. $7 \mathrm{C}$

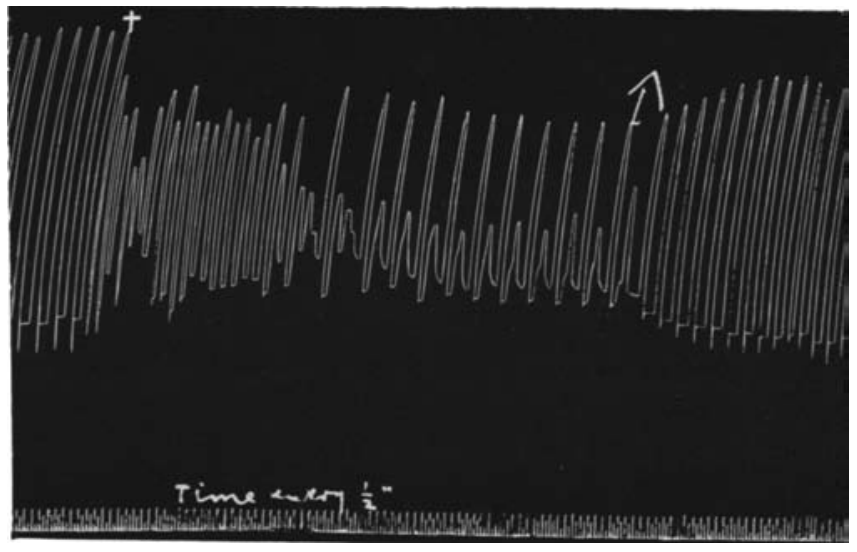

FIG. 70

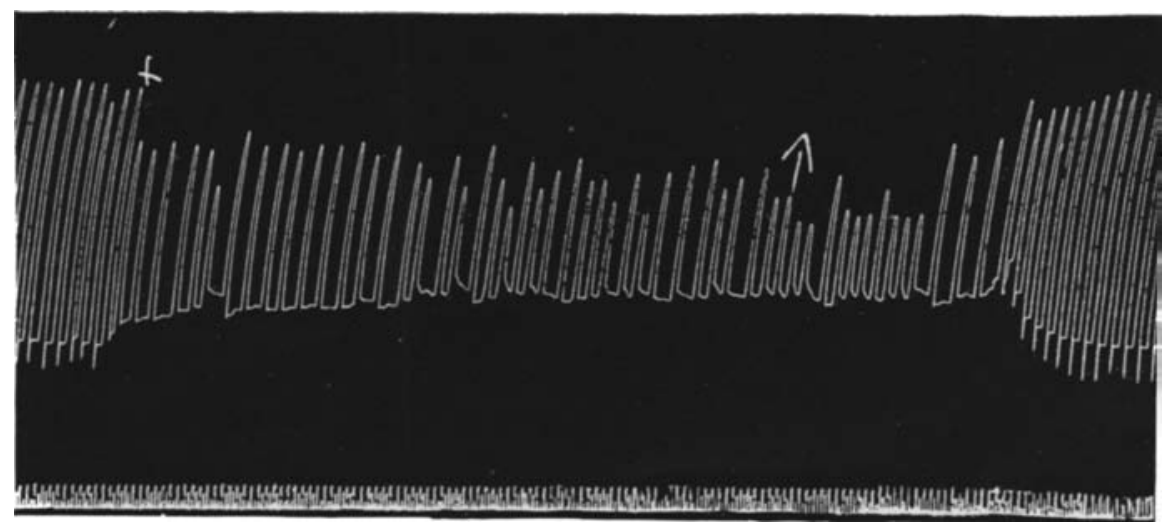

FIG. $7 \mathrm{E}$ 

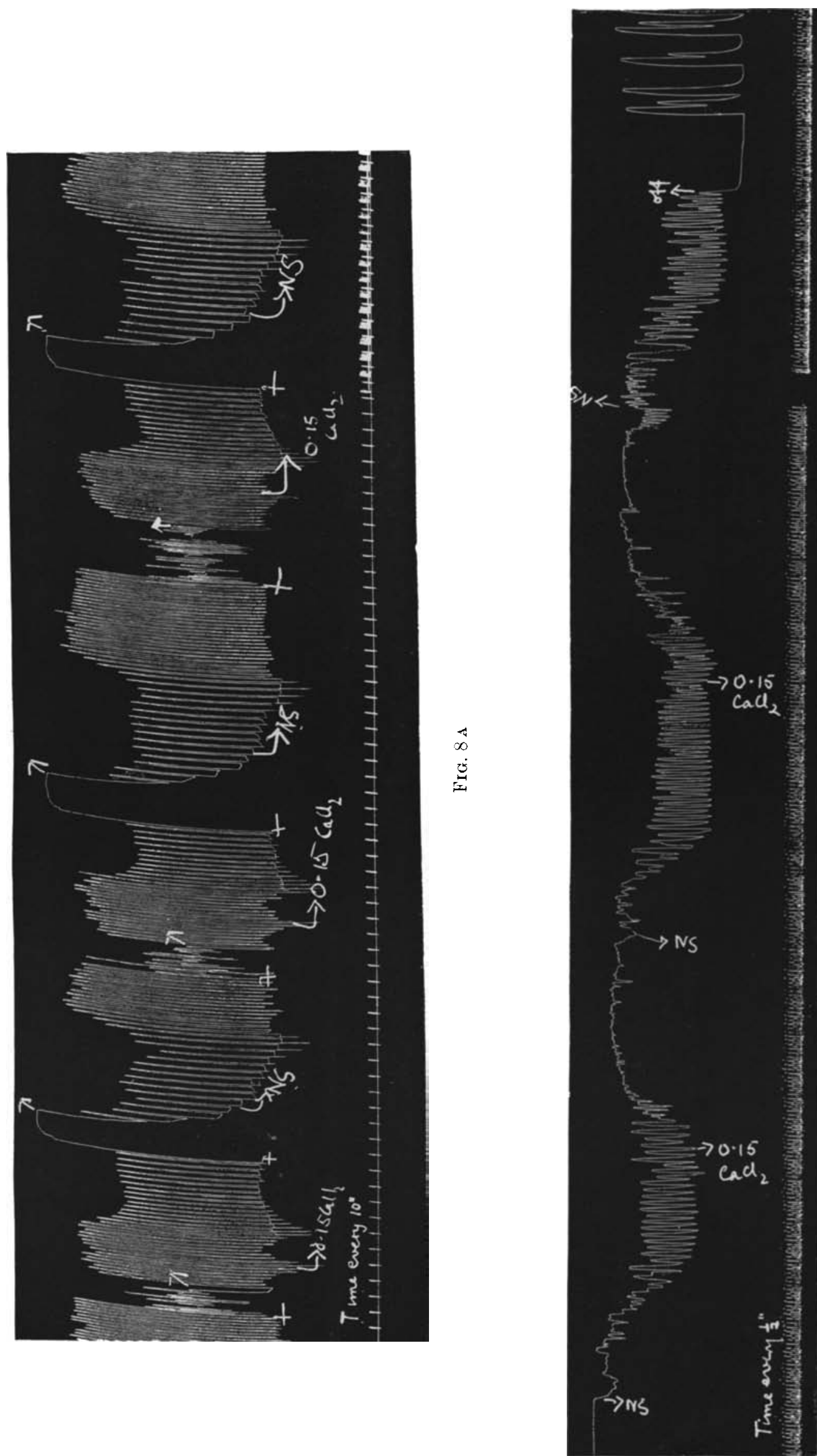

2
$-\infty$
- 